\title{
Editorial: Moving Forward to Respond to Rapid Changes of Computer Science and Technology
}

Since its inaugural issue in 1986, the Journal of Computer Science and Technology (JCST) has been the premier English journal of China Computer Federation $(\mathrm{CCF})$, serving international readers and authors by disseminating scholarly and technical papers under a rigorous review process.

The field of computer science and technology, as well as its knowledge dissemination model, has changed significantly in the past 28 years. As an archival journal covering a wide spectrum of research topics, JCST must adapt to these changes to effectively serve its readers and authors in the field. Starting in the year of 2014, JCST will make two new actions: (1) our editorial board will become more international, and (2) we will publish papers in a more focused way with a theme-based publication approach.

It is my great pleasure to welcome Professor Chung-Laung Liu, a distinguished and senior computer scientist from Tsinghua University (Hsinchu), to our advisory board. Professors Donald Knuth and Andrew Yao will continue serving on the advisory board. Professor Wen-Tsun Wu will retire from the advisory board, and we thank for his guidance and contributions to the journal.

Our plan of implementing a theme-based publication model is motivated by best practices of high-quality international conferences in the computer science fields. Starting from 2015, JCST will introduce a permanent special-section in each issue focusing on one of the six important and mainstream research areas, including Computer Architecture and Systems, Artificial Intelligence and Pattern Recognition, Computer Graphics and Multimedia, Data Management and Data Mining, Software Systems, and Computer Networks and Distributed Computing. Each special section will appear every year in the same issue. Moreover, each of the selected areas and its special section will be edited by a Leading Editor.

I am very pleased to welcome the following six experts to join the JCST Editorial Board as Leading Editors, who are internationally recognized scholars in their fields and enthusiastic volunteers in the professional community.

- Wen-Guang Chen, Leading Editor for Computer Architecture and Systems

- Xi-Lin Chen, Leading Editor for Artificial Intelligence and Pattern Recognition

- Shi-Min Hu, Leading Editor for Computer Graphics and Multimedia

- Jian Pei, Leading Editor for Data Management and Data Mining

- Tao Xie, Leading Editor for Software Systems

- Wen-Wu Zhu, Leading Editor for Computer Networks and Distributed Computing

Each editor's short biography is introduced in this issue.

Finally I would like to thank the strong support and service from our readers, authors and the editorial board. Please continue to keep us informed about your thoughts and suggestions. We will try our best to respond to the needs of the JCST community.

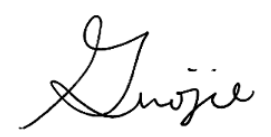

Guo-Jie Li

Editor-in-Chief

Journal of Computer Science and Technology 